\title{
Electroactive Nanocarbon as Novel Label for DNA Analysis ${ }^{+}$
}

\author{
Puay Ching Koh, Hui Qing Shi, Caroline and Alessandra Bonanni * \\ Division of Chemistry \& Biological Chemistry, School of Physical and Mathematical Sciences, Nanyang \\ Technological University, Singapore 637371, Singapore \\ * Correspondence: a.bonanni@ntu.edu.sg \\ + Presented at the 7th International Symposium on Sensor Science, Napoli, Italy, 9-11 May 2019.
}

Published: 31 July 2019

\begin{abstract}
We use here a carbon nanomaterial as the electroactive label for the development of a lowcost and easy-to-miniaturize non-optical polymerase chain reaction (PCR). To this aim, graphene oxide nanocolloids (GONCs) were first conjugated to the DNA primers either by physical adsorption or by the formation of a covalent bond. Subsequently, GONC-modified primers were hybridized to their complementary counterpart to obtain GONC-modified double stranded DNA (GONC-dsDNA). The electrochemical signal, due to the reduction of the electrochemically reducible oxygen functionalities on GONC surface was then measured and correlated to the concertation of GONC-dsDNA.
\end{abstract}

Keywords: electroactive nanocarbon; nano-graphene oxide; DNA analysis; electrochemistry

\section{Introduction}

Among the several chemically modified graphene materials, nano-graphene oxide has attracted vast attention from many research fields owing to its exceptional properties (eg. good conductivity, high surface area, possibility of functionalization, intrinsic electroactivity and fluorescence properties) that it possesses [1-6]. Nano-graphene oxide material consists of highly oxidized twodimensional monoatomic sheets of large surface area, with different oxygen functionalities namely epoxy, peroxy, hydroxyl, carbonyl, and carboxyl groups [1,5]. Existing studies show that graphene oxide possesses an intrinsic electroactivity due to the presence of electrochemically reducible oxygen functionalities on its surface [6]. Specifically, a significant reduction peak can be observed at around $-1.2 \mathrm{~V}$ when the potential is catholically scanned. In this work, we employ graphene oxide nanocolloid (GONC) materials as a label for DNA detection. The abundance of electrochemically reducible oxygen containing groups (OCGs) on the material surface, together with the nanoscale size of the colloids make GONC the ideal material for cost-effective and sensitive labelling.

\section{Materials and Methods}

Graphene Oxide Nanocolloids (GONC), sodium chloride, sodium diphosphate basic monohydrate, $N$-(3-dimethylaminopropyl)- $N$ '-ethylcarbodiimide hydrochloride (EDC), 2-(N-Morpholino) ethanesulfonic acid (MES), N-hydroxysulfosuccinimide sodium (sulfo-NHS), were obtained from Sigma Aldrich (Singapore). DNA oligonucleotides used in this work were purchased from Integrated DNA Technologies, Inc. (Singapore) and their sequences were the following: 5'-GCTCC TACAAATGCCATCA-3' (probe); 5'-TGATGGCATTTGTAGGAGC-3' (target); for the covalent conjugation with GONCs, amino-modification of DNA sequences $5^{\prime}$-end has been carried out. Covalent modification of DNA sequences with GONC has been carried out according to previous 
literature [7]. A similar protocol has been followed for the physical adsorption protocol by skipping the covalent conjugation step. Calibration curves of increasing concentrations of GONC $(0.01 \mathrm{mg} / \mathrm{mL}-1 \mathrm{mg} / \mathrm{mL})$ and GONC-DNA conjugates $(0.0125 \mathrm{mg} / \mathrm{mL}-0.2 \mathrm{mg} / \mathrm{mL})$ were performed by dropcasting $3 \mu \mathrm{L}$ of the sample on the electrode surface and letting it dry before measuring the electrochemical signal. Hybridisation of DNA primer sequences was conducted by incubating the GONC modified DNA sequences with the complementary target at a concentration of $0.48 \mu \mathrm{M}$, at $42^{\circ} \mathrm{C}$ for 40 minutes. After centrifugation, three washing steps were carried out in PBS buffer ( $\mathrm{pH} 7.4)$.

\section{Results}

As part of material characterization, cyclic voltammetry was performed to investigate the presence of OCGs on GONC surface and the intrinsic electroactivity of the material. As shown in Figure 1, a significant reduction peak at about $1.15 \mathrm{~V}$ was observed during the cathodic scan of GONC, which arose from the reduction of electrochemically active OCGs present on GONC surface. The obtained reduction signal confirmed the suitability of the material for the current study.

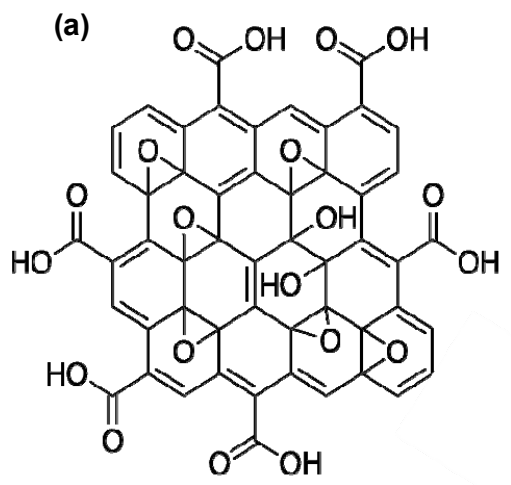

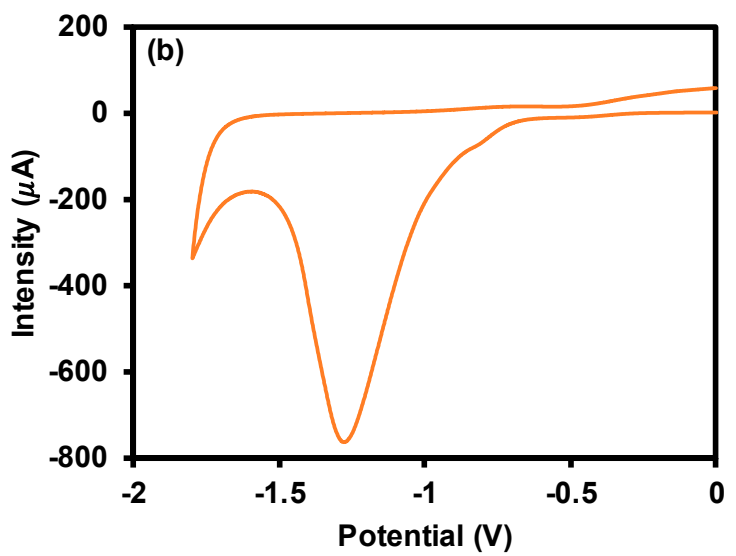

Figure 1. Graphene oxide nanocolloid (GONC) structure (a) and electrochemical reduction peak (b). Conditions: scan rate $50 \mathrm{mV}$; $0.1 \mathrm{M}$ PBS, pH 7.0.

Moving on, GONC material was conjugated to specific DNA sequences following two different protocols namely physical adsorption and covalent conjugation. After which, the GONC modified DNA probes were hybridized with the corresponding complementary target sequences in order to obtain a GONC-dsDNA conjugate. The electrochemical signal obtained from different concentrations of the GONC-dsDNA conjugates synthetized by following the two conjugation protocols was then measured and compared with that obtained from the bare GONCs. Results from this study are shown in Figure 2. Figure 2a depicts the calibration curve that shows the peak heights obtained by differential pulse voltammetry (DPV) measurements for increasing concentrations of GONCs. Figure $2 \mathrm{~b}$ shows the peak heights from the reduction peaks for increasing concentrations of GONC-dsDNA conjugate obtained with the physical adsorption protocol, while Figure $2 \mathrm{c}$ shows the calibration curve obtained by increasing concentrations of GONC-dsDNA conjugate form the covalent conjugation. 

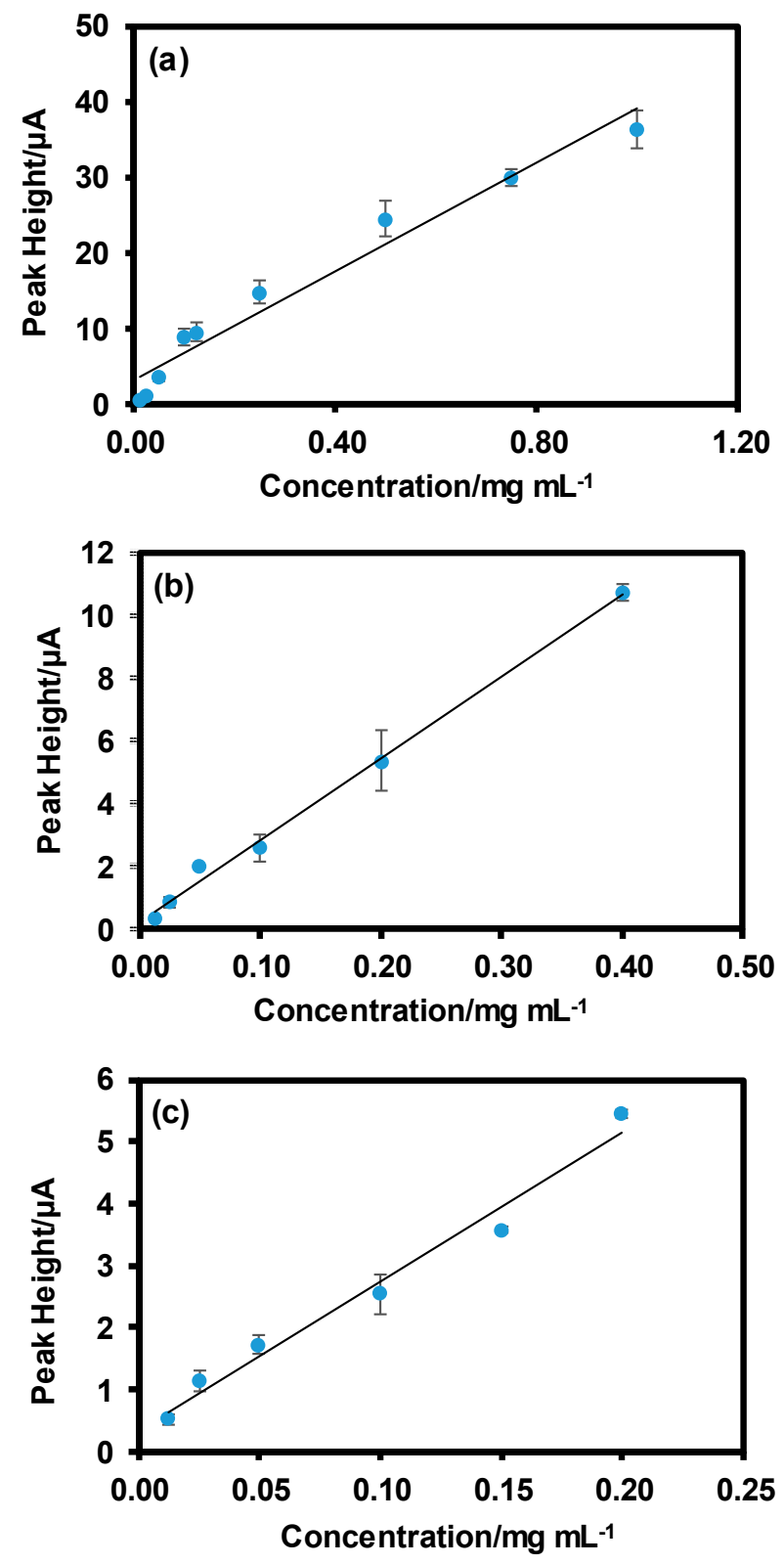

Figure 2. Calibration curve depicting the signal intensity versus concentration for: (a) Bare GONC material; (b) GONC-dsDNA conjugate obtained by physical adsorption; (c) GONC-dsDNA conjugate obtained by covalent conjugation.

\section{Discussion}

In this preliminary study we showed how electrochemically active nano-graphene oxide material could be successfully employed for DNA labelling. As shown from the calibration curves depicted in Figure 2, GONC material maintains a significant electroactivity after the conjugation with the DNA probes and hybridization with complementary targets, allowing the final quantitative analysis of DNA. This system could be eventually integrated in a PCR protocol, with the use of GONC-modified DNA primers that would allow an electrochemical detection and provide a costeffective alternative to the current optical PCR. 
Acknowledgments: The authors gratefully acknowledge a Ministry of Education (MOE), AcRF Tier 1 grant (Reference No: RG18/17) for the funding of this research.

Conflicts of Interest: The authors declare no conflict of interest. The funders had no role in the design of the study; in the collection, analyses, or interpretation of data; in the writing of the manuscript, or in the decision to publish the results.

\section{References}

1. Giuliodori, A. M.; Brandi, A.; Kotla, S.; Perrozzi, F.; Gunnella, R.; Ottaviano, L.; Spurio, R.; Fabbretti, A. Development of a graphene oxide-based assay for the sequence-specific detection of double-stranded DNA molecules. PLoS ONE 2017, 12, e0183952.

2. Cheng, Z.X.; Bonanni, A. All-in-One: Electroactive Nanocarbon as Simultaneous Platform and Label for Single-Step Biosensing. Chem. A Eur. J. 2018, 24, 6380-6385.

3. Toh, S.Y.; Loh, K.S.; Kamarudin, S.K.; Daud, W.R.W. Graphene production via electrochemical reduction of graphene oxide: Synthesis and characterisation. Chem. Eng. J. 2014, 251, 422-434.

4. Ambrosi, A.; Chua, C.K.; Latiff, N.M.; Loo, A.H.; Wong, C.H.A.; Eng, A.Y.S.; Bonanni, A.; Pumera, M. Graphene and Its Electrochemistry-An Update. Chem. Soc. Rev. 2016, 45, 2458-2493.

5. Bonanni, A.; Pumera, M. Graphene Platform for Hairpin-DNA-Based Impedimetric Genosensing. ACS Nano 2011, 5, 2356-2361.

6. Pumera, M. Graphene-based nanomaterials and their electrochemistry. Chem. Soc. Rev. 2010, 39, $4146-4157$.

7. Wang, S.; Zhang, Y.; Pang, G.; Zhang, Y.; Guo, S. Tuning the Aggregation/Disaggregation Behavior of Graphene Quantum Dots by Structure-Switching Aptamer for High-Sensitivity Fluorescent Ochratoxin A Sensor. Anal. Chem. 2017, 89, 1704-1709.

(C) 2019 by the authors. Licensee MDPI, Basel, Switzerland. This article is an open access article distributed under the terms and conditions of the Creative Commons Attribution (CC BY) license (http://creativecommons.org/licenses/by/4.0/). 